\title{
The relationship between major depressive disorder and employment status
}

\author{
Roger C $\underline{\text { Ho }}^{1,2_{F R C P S y c h}}$, Bach X Tran ${ }^{3,4}{ }_{P h D}$, Roger S $\underline{\text { McIntyre }}^{5,6,7,8}{ }_{F R C P C}$
}

Major depressive disorder is associated with executive dysfunction that includes impairment in problemsolving and decision-making, ${ }^{1}$ as well as with occupational impairment. ${ }^{2}$ One of the challenges during the COVID-19 pandemic has been to maintain employment status. It has been reported that employment status is associated with measures of quality of life as well as measures of depression. ${ }^{3}$ Additionally, adverse employment histories in terms of recurrent job discontinuity are associated with an increased risk of self-reported depression in the general population. ${ }^{4}$ Conversely, it has been reported that employment may be a protective factor against postpartum depression in working mothers. ${ }^{5}$ It has been separately reported that unemployed persons with depression-associated disabilities demonstrate decreased workplace function when compared to employed controls. ${ }^{6}$ In addition to unemployment, underemployment has also been associated with an increased risk for depression. ${ }^{7}$ For persons who are unemployed, poor social support, financial hardship and loss of personal control were identified as important mediators of the association between unemployment and depression. ${ }^{7} \mathrm{~A}$ separate body of literature also indicates that persons who are employed exhibit greater response rates with antidepressant treatments. ${ }^{8}$

In contrast to unemployment, employment has been reported to be associated with improved measures of mental health. It has also been reported that employed persons experiencing depression exhibit greater functional improvement in cognition and interpersonal interaction, perhaps reflecting behavioural activation and/or the impact of interpersonal support offered by the workplace setting. ${ }^{6}$ For many persons, the workplace environment is the principal source of their interpersonal connectedness and friendship, and the loss thereof is associated with increased measures of loneliness. ${ }^{9}$ In a separate longitudinal study conducted in the US, employment at baseline was associated with lower levels of depression at baseline and less severe longitudinal course of depression across 20 years of observation. ${ }^{10}$ In the US, treatment-resistant depression, even in comparison to major depressive disorder (MDD), poses a significant direct and indirect cost burden to US employers and is associated with higher rates of employment status change. ${ }^{11}$

Research in Asian countries regarding the relationship between employment status and mental health has been limited. ${ }^{12}$ A recent study conducted in South Korea revealed that Koreans with precarious employment reported a greater prevalence of depression compared to those with permanent employment. ${ }^{12}$ Koreans who lost their vocational status reported the highest depression scores compared to those who maintained their workability and employment. ${ }^{13}$ Furthermore, Korean men working day shifts and women working night shifts in precarious employment were more likely to be depressed than those with permanent employment. ${ }^{12}$ In Japan, it was reported that lower income and unemployment were associated with a higher prevalence of depression and greater probability of receiving antidepressant treatment. ${ }^{14}$ In Singapore, a separate study reported that $50 \%$ of indirect costs for managing MDD were associated with loss of productivity and unemployment. ${ }^{15}$

In this issue of the Annals, Sambasivam et al. published a study on the moderating effect of employment status on the relationship between lifetime MDD and positive mental health (PMH). ${ }^{16}$ This study demonstrated that unemployed Singapore residents reported lower $\mathrm{PMH}$ status than individuals who were employed or in other groups, including students, homemakers, retirees and pensioners. Moreover, it has been reported that Singapore residents with poor mental health have lower economic activity, lower earnings, unstable employment and financial insecurity. Taken together,

\footnotetext{
${ }^{1}$ Department of Psychological Medicine, Yong Loo Lin School of Medicine, National University of Singapore, Singapore

${ }^{2}$ Institute of Health Innovation and Technology (iHealthtech), National University of Singapore, Singapore

${ }^{3}$ Institute for Preventive Medicine and Public Health, Hanoi Medical University, Hanoi, Vietnam

${ }^{4}$ Bloomberg School of Public Health, Johns Hopkins University, Baltimore, MD, US

${ }^{5}$ Department of Psychiatry, University of Toronto, Toronto, Canada

${ }^{6}$ Brain and Cognition Discovery Foundation, Toronto, Canada

${ }^{7}$ Mood Disorders Psychopharmacology Unit, University Health Network, Toronto, Canada

${ }^{8}$ Department of Pharmacology, University of Toronto, Toronto, Canada

Correspondence: Prof Roger C Ho, Department of Psychological Medicine, Yong Loo Lin School of Medicine, National University of Singapore, 10 Medical Dr, Singapore 117597.

Email: pcmrhcm@nus.edu.sg
} 
evidence suggests that mental and economic well-being may have a bidirectional relationship in Singapore.

Sambasivam et al. recommended that healthcare professionals should also consider the employment needs of Singapore residents who suffer from MDD as part of a holistic approach to providing care. ${ }^{16}$ The holistic approach should consist of pharmacological, psychological and social interventions, and where applicable, employment-focused interventions. For pharmacological treatment, previous placebo-controlled and active comparator clinical trials demonstrated the efficacy of selective serotonin re-uptake inhibitors (e.g. fluoxetine, paroxetine and sertraline), serotonin-norepinephrine re-uptake inhibitors (e.g. duloxetine and venlafaxine), and novel antidepressants (e.g. agomelatine and vortioxetine) improve self-reported measures of workplace impairment. ${ }^{17}$

Psychological intervention may be effective for persons with depression by enhancing productivity and improving employment outcomes. During the COVID-19 pandemic, many people need to work from home, and online psychological interventions such as internet-based cognitive behaviour therapy can provide mental health support to workers who work from home, and reduce the risk of spreading COVID-19.18 Social support is needed for unemployed people to overcome the negative impact of losing employment and workplace capability. From the social perspective, governments and social agencies can launch employment programmes and rehabilitation strategies to help people with depression resume work. ${ }^{6}$

The potentially hazardous effects of COVID-19 on mental health have become an international public health priority. ${ }^{19}$ Further research is required to study the relationship between unemployment related to COVID-19 in some of the most seriously affected workplace sectors and depression.

\section{Disclosure}

Dr Roger $S$ McIntyre has received research grant support from CIHR/GACD/National Natural Science Foundation of China; speaker/ consultation fees from Lundbeck, Janssen, Alkermes, Mitsubishi Tanabe, Purdue, Pfizer, Otsuka, Takeda, Neurocrine, Sunovion, Bausch Health, Novo Nordisk, Kris, Sanofi, Eisai, Intra-Cellular, NewBridge Pharmaceuticals, AbbVie, and ATAI Life Sciences. Dr Roger S McIntyre is a CEO of Braxia Scientific Corp.

\section{REFERENCES}

1. Husain SF, McIntyre RS, Tang TB, et al. Functional near-infrared spectroscopy during the verbal fluency task of English-Speaking adults with mood disorders: A preliminary study. J Clin Neurosci 2021;94:94-101

2. McIntyre RS, Cha DS, Soczynska JK, et al. Cognitive deficits and functional outcomes in major depressive disorder: determinants, substrates, and treatment interventions. Depress Anxiety 2013; 30:515-27.

3. Wyndaele JJ. Challenges of today: at one side employment important for quality of life and to avoid depression; at the other side a worldwide economic crisis. Spinal Cord 2013;51:259.

4. Hoven H, Wahrendorf M, Goldberg M, et al. Adverse employment histories, work stress and self-reported depression in the French CONSTANCES study. Eur J Public Health 2021;31:1230-6.

5. Lewis BA, Billing L, Schuver K, et al. The relationship between employment status and depression symptomatology among women at risk for postpartum depression. Womens Health (Lond) 2017;13:3-9.

6. Chiang YC, Liou TH, Lee HC, et al. Using WHODAS 2.0 to Assess Functional Impairment in People with Depression: Should Employment Receive More Attention? Int J Environ Res Public Health 2021;18:4552.

7. Crowe L, Butterworth P. The role of financial hardship, mastery and social support in the association between employment status and depression: results from an Australian longitudinal cohort study. BMJ Open 2016;6:e009834.

8. McIntyre RS, Florea I, Tonnoir B, et al. Efficacy of Vortioxetine on Cognitive Functioning in Working Patients With Major Depressive Disorder. J Clin Psychiatry 2017;78:115-21.

9. Park C, Majeed A, Gill H, et al. The Effect of Loneliness on Distinct Health Outcomes: A Comprehensive Review and MetaAnalysis. Psychiatry Res 2020;294:113514.

10. Heinz AJ, Meffert BN, Halvorson MA, et al. Employment characteristics, work environment, and the course of depression over 23 years: Does employment help foster resilience? Depress Anxiety 2018;35:861-7.

11. Amos TB, Tandon N, Lefebvre P, et al. Direct and Indirect Cost Burden and Change of Employment Status in Treatment-Resistant Depression: A Matched-Cohort Study Using a US Commercial Claims Database. J Clin Psychiatry 2018;79:17m11725.

12. Oh JW, Park JY, Lee S. Association between employment stability and depression as moderated by gender among South Korean employees. J Affect Disord 2022;298(Part A):308-15.

13. Lee SA, Ju YJ, Han KT, et al. The association between loss of work ability and depression: a focus on employment status. Int Arch Occup Environ Health 2017;90:109-16.

14. Fukuda Y, Hiyoshi A. Influences of income and employment on psychological distress and depression treatment in Japanese adults. Environ Health Prev Med 2012;17:10-7.

15. Ho RC, Mak KK, Chua AN, et al. The effect of severity of depressive disorder on economic burden in a university hospital in Singapore. Expert Rev Pharmacoecon Outcomes Res 2013; 13:549-59.

16. Sambasivam R, Vaingankar JA, Abdin E, et al. The moderating effect of employment status on the relationship between lifetime major depression disorder and positive mental health. Ann Acad Med Singap 2022;51:74-86.

17. Lee Y, Rosenblat JD, Lee J, et al. Efficacy of antidepressants on measures of workplace functioning in major depressive disorder: A systematic review. J Affect Disord 2018;227:406-15.

18. Ho CS, Chee CY, Ho RC. Mental Health Strategies to Combat the Psychological Impact of Coronavirus Disease 2019 (COVID-19) Beyond Paranoia and Panic. Ann Acad Med Singap 2020; 49:155-60.

19. Ho RC, Tran BX, McIntyre RS. The impact of COVID-19 pandemic on global mental health: From the general public to healthcare workers. Ann Acad Med Singap 2021;50:198-9. 